\title{
Koppitz e Bender - Sistema de Pontuação Gradual: comparação entre sistemas de avaliação
}

\author{
Avaliação do Teste Bender \\ Ana Paula Porto Noronha \\ Rosângela Maria De Carli Bueri Mattos
}

\section{Resumo}

O objetivo do presente trabalho é comparar os sistemas de correção Koppitz e o B-SPG. Usou-se como amostra 85 protocolos de crianças de seis a dez anos que foram encaminhadas à Clínica-Escola de Psicologia de um centro universitário do sul de Minas Gerais. Os resultados evidenciam que os dois sistemas medem construtos similares, pois houve correlação positiva e significativa entre os escores de ambos. As correlações foram positivas e significativas com a idade, porém o sistema B-SPG obteve melhor condição de agrupamento. Em relação às queixas somente as dificuldades escolares conseguiram correlação positiva significativa para ambos os sistemas. A comparação dos critérios indica que os sistemas possuem sensibilidade para captar a imaturidade visomotora, com maior destaque ao B-SPG por possuir capacidade de identificar a qualidade do erro.

Palavras-chave: Bender; Sistema B-SPG; Maturidade visomotora.

\section{Koppitz e Bender - Sistema de Pontuação Gradual: comparison between systems of evaluation}

\begin{abstract}
The objective of the present work is to compare the systems of Koppitz correction and the B-SPG. It was used as sample 85 protocols of six children about ten years old that had been directed to the Clinic-School of Psychology of an University Center of the South of Minas Gerais. The results evidence that two systems measure similar construct, therefore positive and significant correlations were found between the scores of both. The correlations had been positive and significant with the age, however SPG system got grouping condition better. In relation to the complaints the pertaining to school difficulties had only obtained significant positive correlation for both the systems. The comparison of the criteria indicates that the systems there is sensitivity to catch the visomotor immaturity, with bigger prominence to the B-SPG because there is capacity to identify the mistake's quality. Key Words: Bender; B-SPG System; Visomotor maturity.

\section{Koppitz y Bender - Sistema de Puntuación Gradual: comparación entre sistemas de evaluación}

\section{Resumen}

El objetivo de este trabajo es comparar los sistemas de corrección de Koppitz y el B-SPG. Se utilizó como muestra 85 protocolos de niños de seis a diez años que fueron encaminados a la Clínica-Escuela de Psicología de un centro universitario del sur de Minas Gerais. Los resultados mostraron que los dos sistemas miden constructos similares, pues hubo correlación positiva y significativa entre las puntuaciones de ambos. Las correlaciones fueron positivas y significativas con la edad, pese a el sistema B-SPG obtener una mejor condición de agrupamiento. En relación a las quejas solamente las dificultades escolares consiguieron correlación positiva significativa para ambos sistemas. La comparación de los criterios indica que los sistemas poseen sensibilidad para captar la inmadurez visomotora, con mayor destaque al B-SPG por poseer capacidad de identificar la calidad del error.

Palabras-Clave: Bender; Sistema B-SPG; Madurez visomotora. 


\section{Introdução}

A publicação em 1938 da monografia Visual Motor Gestalt Test and its Clinical Use de Lauretta Bender, marca o começo do Bender Visual-Motor Gestalt Test, um dos instrumentos psicométricos mais usados em investigações e pesquisados sobre testes (Buckley, 1978; Noronha \& cols., 2002). O Bender foi definido como um teste de maturidade da performance da função gestáltica visomotora para crianças de 4 a II anos, capaz de fazer estimativas do desenvolvimento visomotor. Este é um conceito fundamental a ser associado com várias outras funções da inteligência, tais como a percepção visual, habilidade motora manual, memória, conceitos espaciais e temporais e organização (Koppitz, 1989).

A aceitação do material proposto por Bender, além de ter sido imediata, suscitou derivações de vários outros sistemas de avaliação com diversificações tanto das faixas etárias como de outras áreas de funcionamento e habilidades do indivíduo. No rastreamento de sua história, Piotrowski (1995) encontrou pesquisas que evidenciaram, com menos de dez anos de existência, o Bender, classificado como o quarto teste mais utilizado nos Estados Unidos, ficando atrás apenas do Rorschach, do Teste da Figura Humana e do Teste de Apercepção Temática, respectivamente.

Entretanto, apesar de muito popular, este teste ainda hoje parece não ter um conjunto uniforme de critério de avaliação consistentemente usado pelos clínicos que não gere algum desacordo e dúvidas de confiabilidade (Piotrowski, 1995). Depois de quase setenta anos de existência, o Bender continua suscitando indagações acerca de sua aplicabilidade tanto das áreas capazes de mensurar como dos sistemas propostos de avaliação. Brannigan e Decker (2003) citam pesquisa que fizeram junto a American Psychological Association (APA) em 2002, na qual constataram cerca de 1300 referências a pesquisas feitas com o Bender, sendo que os sistemas mais citados foram os de Hutt, Pascal e Suttel, embora a maioria fosse em relação ao sistema de pontuação de Koppitz.

Chamado de The Developmental Bender Scoring System e criado em 1963 por Elizabeth Koppitz ele provê três diferentes análises de um protocolo e obtêm a avaliação da maturidade perceptiva, índices de possível deteorização neurológica e o ajustamento emocional (Koppitz, 1989). O sistema de pontuação compreende quatro categorias de erros na reprodução dos desenhos, sendo distorção, rotação, integração e perseveração. Os itens de pontuação foram selecionados a partir de pesquisa com 77 crianças de 6 anos e 4 meses aos 10 anos e 8 meses de inteligência normal divididos em grupos contrastantes, sendo um de $4 \mathrm{I}$ com melhores rendimentos e bom ajustamento e o outro de $36 \mathrm{com}$ baixo rendimento escolar. Sua hipótese era que o aluno iniciante com percepção visomotora bem desenvolvida teria, provavelmente, um bom rendimento escolar nos primeiros anos do ensino fundamental, diferentemente do aluno com imaturidade visomotora, cuja probabilidade seria de dificuldade nas tarefas escolares.

Embora o sistema de pontuação desenvolvido por Koppitz seja citado como o mais amplamente pesquisado e usado dos sistemas de correção existentes do teste Bender (Brannigan \& Bruner, 1989), várias pesquisas estrangeiras (Buckey, 1978; Mitchell-Burns, 2000; Levin \& González, 2004; Chan, 2000) e nacionais (Machado, 1978; Moura, 1982; La Puente \& Maciel Jr., 1984; Pinelli Jr. \& Pasquali, 1990; Bandeira \& Hutz, 1994) para elencar algumas, têm apontado para a fragilidade do sistema de pontuação de Koppitz no que diz respeito a discrepâncias de resultados. A seguir, são citados alguns trabalhos que questionam os parâmetros psicométricos desse sistema de avaliação.

Para faixa etária infantil, em um extenso levantamento bibliográfico, no âmbito da predição, Lesiak (1984) fez revisão em 32 pesquisas de estudos publicadas em três seguimentos de capacidade de leitura, sendo a utilidade do Bender, em diversos sistemas de correção, como preditor de leitura, a relação entre o Bender e a realização de leitura medidas concomitantemente e a capacidade do Bender para diferenciar entre bons e maus leitores. $O$ autor concluiu que o Bender não indica uma diferença significativa entre bons e maus leitores, assim como não prediz com segurança êxito na leitura, portanto não é justificável a inclusão dele numa bateria de diagnóstico de leitura. 
$\mathrm{Na}$ mesma linha de escolaridade, com o objetivo de examinar se a habilidade visomotora é capaz de predizer o rendimento escolar de crianças, Chan (2000) fez um estudo com 748 crianças chinesas de quatro a oito anos que cursavam pré-escola e ensino fundamental com nível socioeconômico médio comparando os sistemas Koppitz e o Qualitativo para a modalidade Modificada do Teste Bender. Como critério de rendimento escolar foram utilizadas as médias de inglês, de chinês e de matemática. $A$ análise de variância demonstrou que o sistema qualitativo é melhor que o Koppitz na predição do rendimento acadêmico.

$\mathrm{Na}$ área de comprometimentos orgânicos e funcionais associados, tem-se o estudo de validade do sistema Koppitz de Nyfield e Patalano (1998). Obtiveram parcial confirmação da hipótese de que há dois subtipos de erros nesse sistema de pontuação, aqueles que são válidos indicadores de prejuízo orgânico (categoria $A$ ) e outros que não aparecem com a função específica de fator emocional (categoria B). O teste Bender foi aplicado em 46 sujeitos do sexo masculino com Transtorno de Déficit de Atenção e Hiperatividade (TDAH), distúrbio de conduta e distúrbio de oposição. Fizeram duas aplicações, sendo a primeira na seqüência proposta pelo teste e a segunda na ordem inversa. Hipotetizaram e confirmaram que ocorreriam mais erros nas figuras apresentadas no final do que as iniciais na seqüência para os sujeitos da categoria $B$ do que para categoria $A$.

Também para faixa etária de adultos são encontrados resultados que não confirmam a validade do sistema Koppitz. Pesquisa realizada por Dixon (1998) com mulheres com retardo mental não obteve validade concorrente para os indicadores emocionais quando comparados com os inventários Reiss Screen e o Client and Agency Planning, os quais são amplamente usados e com grande aceitação. Outra pesquisa que demonstrou que os dados normativos do sistema Koppitz são frágeis para captar distúrbio perceptivo visomotor foi realizado por Raggio (1999) com préadolescentes com diagnóstico de TDAH.

Em âmbito nacional, há quase trinta anos, já existem críticas quanto ao uso do Bender, visto que os resultados de pesquisa apontam para a restrita capacidade de discriminação entre crianças com alto e baixo rendimento escolar, com e sem problemas emocionais e de conduta. Esta pesquisa trabalhou com 77 crianças de idade escolar entre seis e dez anos sem indícios de baixo rendimento intelectual. Machado (1978) concluiu que o Bender não foi útil para avaliar significativamente as crianças com problemas escolares e de aprendizagem, uma vez que todas obtiveram pontuações.

Sob o ponto de vista de La Puente e Maciel Jr. (I984) uma avaliação dupla, na qual seriam associados os aspectos quantitativos e os qualitativos, teria maior força em evidenciar reais disfunções visomotoras e discriminar pontos de corte para diagnósticos. Por outro lado, é o importante estudo de Pinelli Jr. e Pasquali (I991/1992) que aponta para alguns itens do sistema Koppitz, não discriminativos, enquanto outros são insuficientes para uma pontuação significativa que contribua para um escore total sensível para diagnóstico.

Em pesquisa realizada com crianças de 5 a 15 anos em Niterói, Brito e Santos (1996) confirmam que o sistema Koppitz não é bom indicador de sexo e idade. A pesquisa foi feita com 398 crianças, sendo 199 meninos e 199 meninas de escola pública, com mensuração da velocidade, do desempenho e de distúrbios emocionais em grupos de crianças normais e estressadas. Apenas a idade foi significativa para o desempenho. Para a rapidez, não houve diferença significativa nos erros apresentados por crianças mais velhas e mais novas. Quanto aos indicadores emocionais, embora os meninos tivessem tido maior pontuação que as meninas, estes apresentaram pontuação significativa nos dois grupos.

Ainda nesta mesma linha de avaliação dos critérios propostos por Koppitz, duas pesquisas evidenciam o restrito valor discriminatório das pontuações. A primeira foi quanto à ineficiência para discriminar o caráter evolutivo por meio do critério de distorção da forma. O estudo realizado por Sisto, Noronha e Santos (2004a) com 3I4 crianças das primeiras séries do ensino fundamental de ambos os sexos com idades de 7 a 10 anos, não mostrou indicadores que possibilitassem diferenciações entre as idades. Estes 
mesmos autores, em outro estudo, observaram que o critério de integração também apresenta problemas na avaliação da maturidade visomotora. Apenas dois grupos etários puderam ser formados pelo teste de Tukey, o que demonstra a incapacidade das avaliações das figuras oferecer uma pontuação individual ou total que diferencie adequadamente as diversas idades (Sisto, Noronha \& Santos, 2004b).

Pode-se observar, a partir do exposto, que o sistema de pontuação de Koppitz, pode-se evidenciar que haja discordância quanto à sua capacidade de avaliar a maturidade visomotora nos diversos seguimentos de características das amostras, sejam elas de idade, distúrbios ou disfunções. O fato de gerar tanto interesse parece estar ligado por se apresentar como uma avaliação de fácil compreensão e execução, além de ter tido uma proposta abrangente de áreas nas quais ele poderia ser empregado como instrumento de medida.

Várias pesquisas estudaram o Bender para várias funções, tais como detectar a maturidade para a aprendizagem com populações diversas (Mazzeschi \& Liz, 1999), para predizer desempenho escolar (Gemignani \& Chiari, 2000), para diagnosticar problemas de leitura e de aprendizagem (Brannigan \& Brunner, 1989), para avaliar dificuldades emocionais (Clawson, 1980) e para diagnosticar lesão cerebral (Ferrante, 2004). Em sua versão final, Koppitz apresentou as distorções no Bender que refletem primariamente a distorção perceptiva ou a imaturidade e as que refletem fatores e atitudes emocionais (Koppitz, 1989).

Por apresentar esta variedade de estudos com o Bender, o sistema de pontuação Koppitz é sem dúvida, um importante método de avaliação psicométrica, e, portanto merecedor de maiores investimentos em pesquisas. Foi nesse contexto que se objetivou 0 interesse do presente estudo ao comparar o sistema Koppitz com o método denominado de Sistema de Pontuação Gradual (B-SPG) que tem como autores Sisto, Noronha e Santos (2006). A aprovação do método B-SPG pelo SATEPSI (Sistema de Avaliação de Testes Psicológicos) do Conselho Federal de Psicologia (CFP, 2006), indica que ele apresenta todos os requisitos necessários para que seja utilizado com segurança na avaliação psicológica, resguardando todos os preceitos da ética profissional.

Nesse sistema, a proposta é diferenciar a gravidade do erro cometido pelo sujeito, uma vez que haverá atribuição de maior pontuação quando a distorção na reprodução da figura for mais acentuada. Desta forma, não se avalia apenas presença ou ausência de erro como no sistema Koppitz, mas também a intensidade deste, possibilitando uma discriminação melhor por figura e por pontuação total.B-SPG para a amostra brasileira.

Vários são os estímulos na busca de dados para esses sistemas de correção. Em primeiro lugar, o sistema Koppitz tem grande importância para a avaliação psicológica. Contudo, a maior motivação para o presente estudo, reside na preocupação de diversos autores (Machado, 1978; Pinelli Jr. \& Pasquali, 1991; Bandeira \& Hutz, 1994; Brito \& Santos, 1996; Sisto, Bueno \& Rueda, 2003) aos parâmetros para uso na população brasileira. Soma-se aos fatos expostos, a necessidade constante de atualização dos parâmetros psicométricos. Dentro dessa perspectiva, o sistema B-SPG representa um ganho para a avaliação psicológica, visto que além de apresentar uma padronização dentro dos requisitos exigidos, é fruto de pesquisas nacionais. $\mathrm{O}$ objetivo do presente estudo é a comparação entre os sistemas de correções Koppitz e o B-SPG.

\section{Método}

\section{Participantes}

Os dados utilizados nesta pesquisa fazem parte de um banco de dados de uma Clínica-Escola de Psicologia do Estado de Minas Gerais instalada em um Centro Universitário particular. $O$ material consta de 85 protocolos de crianças que passaram por psicodiagnótico de 2001 a 2003, período em que foi utilizado o Bender como um dos instrumentos de avaliação. A amostra é composta de crianças de 6 a 10 anos, sendo $78,8 \%$ meninos e $21,2 \%$ meninas. A média de idade do total de crianças foi de 8,2 anos (DP I,34). A Tabela I mostra a distribuição dos sujeitos de acordo com a série, idade e sexo. 
Tabela I. Distribuição das porcentagens dos participantes de acordo com série, idade e sexo

\begin{tabular}{|c|c|c|c|c|c|c|c|c|c|c|c|c|}
\hline \multirow{2}{*}{ Série } & \multicolumn{2}{|c|}{6} & \multicolumn{2}{|c|}{7} & \multicolumn{2}{|c|}{8} & \multicolumn{2}{|c|}{9} & \multicolumn{2}{|c|}{10} & \multicolumn{2}{|c|}{ Total série /sexo } \\
\hline & M & $\mathrm{F}$ & M & $\mathrm{F}$ & M & $\mathrm{F}$ & M & $\mathrm{F}$ & M & $\mathrm{F}$ & M & $\mathrm{F}$ \\
\hline Pré & 11,9 & 5,6 & --- & --- & --- & --- & --- & --- & --- & --- & 11,9 & 5,6 \\
\hline $1^{\mathrm{a}}$ & 1,5 & 22,2 & 9,0 & 5,6 & 3,0 & --- & 1,5 & --- & 1,5 & --- & 16,4 & 27,8 \\
\hline $2^{\mathrm{a}}$ & --- & --- & 3,0 & 11,1 & 16,3 & 16,7 & 7,5 & --- & 1,5 & 5,6 & 28,4 & 33,3 \\
\hline $3^{\mathrm{a}}$ & --- & --- & --- & --- & 3,0 & 5,6 & 22,3 & 11,1 & 4,5 & 5,6 & 29,9 & 22,2 \\
\hline $4^{\mathrm{a}}$ & --- & --- & --- & --- & --- & --- & 3,0 & --- & 7,5 & --- & 10,4 & --- \\
\hline $5^{\mathrm{a}}$ & --- & --- & --- & --- & --- & --- & - & --- & 3,0 & 11,1 & 3,0 & 11,1 \\
\hline Total idade/sexo & 13,4 & 27,8 & 12,0 & 16,7 & 22,3 & 22,2 & 34,3 & 11,0 & 18,0 & 22,2 & 100,0 & 100,0 \\
\hline
\end{tabular}

A Tabela I evidencia que, apesar da pequena porcentagem amostral do sexo feminino, sua distribuição apresenta uma menor dispersão nas séries escolares de acordo com a idade, com maior freqüência na ${ }^{a}$ série. A dispersão do sexo masculino se amplia gradualmente de acordo com o aumento da idade, sendo que a maior freqüência se encontra na $3^{\mathrm{a}}$ série com idade de nove anos.

De acordo com os onze tipos de queixas dos participantes, encaminhados ao psicodiagnóstico, é possível evidenciar que as mais freqüentes foram agressividade $(40,0 \%)$ seguida de dificuldade de aprendizagem $(28,2 \%)$, tendo no outro extremo irritabilidade e birra/falta de limites (3,5\% cada) e preocupação familiar ( $1,2 \%)$. As queixas, por demonstrarem que existe uma sintomatologia complementar da mesma área entre elas, foram agrupadas nas categorias comportamento, escolar e emocional de acordo com as dificuldades apresentadas. Para comportamento, composta por agressividade, irritabilidade, hiperatividade e birra/ limites atingiu 59\%; escolar representada pela dificuldade de aprendizagem, obteve $28 \%$ e emocional a qual incluiu as queixas de nervoso/choro, insegurança/ medo e preocupação familiar, com 13\%. A maior ocorrência das queixas é quanto ao comportamento das crianças, a qual supera a porcentagem da somatória dos outros dois tipos (4I\%).

\section{Material}

A partir dos dados coletados no psicodiagnóstico, foram feitos uma Ficha de Informações com dados pessoais para os sujeitos (sexo, idade, escolaridade, queixa primária e secundária) e uma Ficha de Resultados com os resultados das correções do Teste Gestáltico Visomotor de Bender pelo Sistema Koppitz e B-SPG. A pesquisadora revisou a correção já existente pelo método Koppitz e realizou a correção B-SPG (Sisto, Noronha \& Santos, 2006) dos protocolos do Bender. Os resultados foram organizados numa planilha eletrônica para posterior análise descritiva e inferencial dos dados, verificando acertos e erros, além da caracterização dos itens e as correlações entre as variáveis.

O Teste Gestáltico Visomotor de Bender, cujo objetivo é a avaliação percepto-motora, é composto por nove (9) cartões com estímulos gráficos, apresentados aos sujeitos para serem copiados. Nessa pesquisa foram utilizados os métodos de correção da Koppitz e B-SPG. A diferença entre os dois métodos está no critério avaliativo, na forma de pontuação, e na escolha da parte do desenho que será avaliada.

O método Koppitz, na mensuração da maturidade percepto-motora, propõe a correção de quatro critérios avaliativos da produção gráfica do sujeito: distorção da forma, rotação, integração e perseveração. Todas as figuras dos desenhos recebem avaliação. A pontuação é invertida, ou seja, atribuição de um ponto para cada erro cometido, atingindo no máximo 30 pontos. A somatória dos pontos é comparada com uma tabela padrão que fornece a idade visomotora do sujeito. A pesquisa para elaboração das normas, envolveu II 04 crianças de ambos os sexos, 
de 5 anos a 10 anos e 11 meses, nas quais foram aplicadas as nove figuras de forma individual. As crianças eram de 12 escolas públicas, de 46 salas que incluíam do jardim de infância à $5^{\mathrm{a}}$ série, dentre as quais encontravam-se crianças com problemas emocionais, dificuldade de aprendizagem, lesão cerebral e deficiência mental (Koppitz, 1980).

Para avaliar a confiabilidade, foi usado o método do teste-reteste com intervalo de quatro meses cujo resultado do coeficiente de correlação de Graus de Kendall ficou entre 0,55 e 0,66, estatisticamente significativas ao nível de $p<0,00 \mathrm{I}$. A validação foi feita por meio da confrontação de cada item com o desempenho na $\mathrm{I}^{\mathrm{a}}$ e $2^{\mathrm{a}}$ séries, medido no Teste Metropolitano de Prontidão. Participaram 165 alunos, sendo 99 da primeira série ( 59 acima da média e 40 abaixo da média) e 66 da segunda série ( 45 acima da média e $2 \mathrm{I}$ abaixo da média). Os qui-quadrados foram estatisticamente significativos ao nível de $p<0.00$ I (Koppitz, 1989). Os resultados indicam adequação do teste para crianças de 6 a 10 anos.

O método B-SPG considera apenas o critério de distorção da forma. A pontuação também é invertida. Atribui-se uma nota de 0 até 2 , com exceção da figura 6, que pode receber também três pontos, respectivamente de acordo com maior comprometimento da qualidade do desenho, podendo totalizar 21 pontos. Outra diferença é que nas figuras A e oito só uma parte da figura é avaliada e na figura 7 , os hexágonos são pontuados separadamente. A soma total de pontos do sujeito deverá ser comparada com a tabela de percentil e quartil. Esta correção fornece dados comparativos de idade, isto é, se o protocolo é discrepante, se o erro é característico, o ritmo de desenvolvimento representacional da criança. Vários foram os estudos de validade do instrumento. Dentre eles, destaca-se a evidência baseada no desenvolvimento, sendo que a correlação entre idades foi de 0,58 a $0,60(p<0,000)$. Ainda, a idade foi fonte de maior variância entre as idades $[F(4,1048)=155,15]$, além de estatisticamente significativa. Sexo não se mostrou como fonte de variância suficiente para revelar diferenças significativas.

Ainda, quanto aos estudos de validade, Suehiro e Santos (no prelo) realizaram um estudo com 287 crianças, ambos os sexos, entre 7 e 10 anos $(M=8,6$; $D P=0,76)$, das segundas ( $53 \% ; N=152)$ e terceiras séries (47\%; $N=135)$ de escolas públicas e particulares do interior de São Paulo. As crianças da terceira série apresentaram melhor desempenho $(M=7,40)$ quando comparadas às da segunda série $(M=8,92)$, conforme o esperado, pois elas apresentaram menor quantidade de erro. Com base no Teste $t$ de Student, constatou-se diferença estatisticamente significativa entre as séries analisadas.

\section{Procedimento}

Essa pesquisa teve 0 aval da Responsável Técnica da Clínica Escola por meio da assinatura do Termo de Consentimento e Autorização da Instituição, encaminhado e aceito ao Comitê de Ética com a solicitação da liberação do Termo de Livre Consentimento e Esclarecido pela dificuldade de obtenção deste tempo de coleta das informações do Comitê de Ética para a utilização dos protocolos. Ressalva-se que na Clínica Escola, todos os clientes assinam um Termo de Consentimento para uso dos dados em pesquisas.

\section{Resultados}

Em razão dos objetivos desse estudo, primeiramente são apresentadas as análises das médias e dos desvios padrões dos dois sistemas. Em seguida são efetuadas as porcentagens de cada figura do teste quanto à pontuação dos erros para cada um dos sistemas de correção, oferecendo um panorama da performance visomotora do grupo investigado. A comparação de médias e a prova de Tukey possibilitaram $\circ$ agrupamento das idades. Posteriormente foi investigada a correlação quanto à idade e série entre o B-SPG e o Sistema Koppitz, no sentido de checar a ocorrência de convergência ou divergência entre os dois sistemas de pontuação, que visam à investigação da maturação percepto-motora. Finalmente foi realizada a correlação entre os tipos de queixas e os sistemas de correção.

Cabe destacar, neste momento inicial da apresentação dos resultados, que eles foram elaborados a partir de uma amostra por conveniência de crianças advindas de uma clínica-escola, e que, 
portanto, possuíam uma demanda psicológica constituída por queixas variadas. Assim, as análises só se justificam quando olhadas à luz dessa informação, sendo que qualquer tipo de comparação com outra população deve levar isso em consideração.

Com o objetivo de verificar a ocorrência de dificuldade na percepção visomotora foi feita a comparação de médias e o desvio-padrão dos dois sistemas. Quanto às médias das pontuações totais, o sistema Koppitz atingiu no máximo 21 dos 30 possíveis de serem obtidos, com média 6,86 (DP 4,6I) para o máximo de 21 pontos dos 30 possíveis de serem obtidos. No sistema B-SPG, a média foi 9,79 (DP 5,3I) para 21 pontos, sendo esta a pontuação máxima do sistema de correção. Nota-se que além de atingir uma média maior, o sistema B-SPG usa a extensão total de suas pontuações. Os valores dos dois desvios padrões demonstraram a heterogeneidade da amostra, fato esperado em função dos protocolos serem de clientes psicológicos.

Para verificar as ocorrências de pontuação em cada uma das figuras fez-se a porcentagem e médias com utilização do qui-quadrado para averiguar a relevância das medidas para os dois sistemas de correção, cujos resultados são visualizados na Tabela 2 . Analisando-se os erros na execução de cada figura pela correção Koppitz pode-se constatar que a distorção da forma $(\mathrm{a}+\mathrm{b})$ atingiu as maiores porcentagens em oito das nove figuras em relação aos demais critérios. Nas figuras 7 e 8 , os erros foram os mais freqüentes com $77,7 \%$ de ocorrência cada, seguida da figura $A$ com
$6 I, 2 \%$, a figura 5 com $47,1 \%$, a figura 3 com $44,7 \%$, a figura 6 com $40,0 \%$.

De todos os demais tipos de erros apenas a integração $(a+b)$ na figura 3 atingiu 36,5\% ultrapassando os $34,1 \%$ obtidos pela figura I no quesito distorção da forma. Em geral, o critério rotação foi o menos pontuado, sendo que obteve a menor porcentagem na figura I (I,2\%). Pode-se observar que, em termos da média de porcentagem por critério, a distorção da forma é a mais elevada pela especificidade ( $a$ e $b$ ), como no conjunto $(a+b)$.

Verificando-se a relevância dos quatro critérios e suas subdivisões de correção, notou-se pelos resultados do qui-quadrado que rotação,e integração b e perseveração não atingiram níveis aceitáveis. Os demais $\chi^{2}$ ficaram no nível de significância.

Já ao relacionar os erros na execução de cada figura pela correção B-SPG tem-se uma variação de $92,9 \%$ a $42,3 \%$ de ocorrência, em ordem de hierarquia decrescente das figuras 2, 4, 8, 5, 6 e 7 (média de $a+b), 3, A$ e I. Ao se verificar a relevância das pontuações de correção, os resultados do quiquadrado confirmam a aceitabilidade dos critérios de I e 2 pontos, com exceção do de três pontos para a figura 3 que, por ser atribuído a uma única figura não pode ser feito. Esses dados podem ser observados a seguir na Tabela 3.

Com vistas a verificar até que ponto os sistemas Koppitz e B-SPG se aproximam, usou-se a correlação de Pearson ao nível de significância 0,01 cujo resultado evidencia que há correlação positiva muito

Tabela 2. Distribuição de porcentagens, médias e qui-quadrado dos erros da amostra pela correção sistema Koppitz

\begin{tabular}{lrrrrrrrrrrrr}
\hline & $\mathrm{A}$ & 1 & \multicolumn{1}{c}{2} & \multicolumn{1}{c}{3} & \multicolumn{1}{c}{5} & \multicolumn{1}{c}{5} & 6 & 7 & 8 & Média & $\chi_{\circ}{ }^{2}$ & $\chi^{2}$ \\
Distorção forma a & 53,0 & 34,1 & -- & 44,7 & -- & 47,1 & 31,8 & 11,8 & 11,8 & 33,4 & 48,86 & 12,59 \\
Distorção forma b & 8,2 & -- & -- & -- & -- & -- & 8,2 & 65,9 & 65,9 & 37,1 & 89,85 & 9,84 \\
Rotação & 18,8 & 1,2 & 22,4 & 20,0 & 22,4 & 22,4 & -- & 21,2 & 21,2 & 18,7 & 0,54 & 12,56 \\
Integração a & 21,1 & -- & 29,4 & 34,1 & 29,4 & 9,4 & 7,1 & 28,2 & 28,2 & 23,3 & 29,94 & 14,07 \\
Integração b & -- & -- & -- & 2,4 & -- & 8,2 & -- & -- & -- & 5,3 & 3,17 & 3,84 \\
Perseveração & -- & 21,2 & -- & -- & -- & -- & 31,8 & -- & -- & 25,1 & 2,70 & 5,99 \\
Média total/figura & 25,3 & 18,8 & 25,9 & 25,3 & 25,9 & 21,8 & 19,7 & 31,8 & 31,8 & & & \\
\hline
\end{tabular}


Tabela 3. Distribuição de porcentagens, médias e qui-quadrado dos tipos de erros da amostra pela correção do sistema B-SPG

\begin{tabular}{ccccccccccccccc}
\hline & $\mathrm{A}$ & 1 & 2 & 3 & 4 & 5 & 6 & $7 \mathrm{~A}$ & $7 \mathrm{~B}$ & 8 & Média & \multirow{2}{*}{${ }^{2}$} & \multirow{2}{*}{$\chi^{2}$} \\
\cline { 2 - 11 } & $\%$ & $\%$ & $\%$ & $\%$ & $\%$ & $\%$ & $\%$ & $\%$ & $\%$ & $\%$ & $\%$ & & \\
\hline 0 ponto & 37,6 & 57,6 & 7,1 & 35,3 & 14,1 & 31,8 & 32,9 & 29,4 & 36,5 & 29,4 & 31,2 & 53.44 & 16,92 \\
1 ponto & 49,4 & 24,7 & 69,4 & 30,6 & 58,8 & 41,2 & 31,8 & 43,5 & 28,2 & 36,5 & 41,4 & 44,46 & 16,02 \\
2 pontos & 12,9 & 17,6 & 23,5 & 34,1 & 27,1 & 27,1 & 16,5 & 27,1 & 35,3 & 34,1 & 25,5 & 21,84 & 16,92 \\
3 pontos & -- & --- & -- & -- & -- & -- & 18,8 & -- & -- & -- & $* * * *$ & $* * * *$ & $* * * *$ \\
\hline Total erros & 62,3 & 42,3 & 92,9 & 64,7 & 85,9 & 68,2 & 67,1 & 70,6 & 63,5 & 70,6 & & & \\
\hline
\end{tabular}

***** não é possível fazer o qui-quadrado por ter apenas um elemento

alta entre as pontuações totais das correções, sendo $r=0,82$ e $p=0,000$, conforme representado na Tabela 4. Pode-se dizer que os dois sistemas de avaliação apresentam correlação significante negativa tanto para escolaridade como para idade $(r=-0,69$, $r=-0,6 \mathrm{I} ; r=-0,67, r=-0,53$ para B-SPG e Koppitz respectivamente). Os dois sistemas apresentam capacidades semelhantes para relacionar a série cursada com a pontuação no teste, porém o sistema B-SPG é mais sensível para classificar quanto à relação idade-pontuação no teste. dados evidenciam que a correlação é baixa negativa significativa com as queixas de comportamento $(r=-0 ; 29 . p=0.007 ; r=-0,26 . p=0,016)$ e baixa positiva significativa com escolar $(r=0,28$, $p=0,009 ; r=0,22, p=0,04 I)$. O tipo de queixa emocional não obteve índices significativos de correlação. Os dados podem ser visualizados na Tabela 5.

Para verificar se havia diferença estatisticamente significativa entre as idades e a pontuação dos sistemas de correção do Bender, utilizou-se a

Tabela 4. Coeficiente de Correlação de Pearson e níveis de significância entre as pontuações dos sujeitos no Bender nos sistemas Koppitz e B-SPG, idade e série $(\mathrm{N}=85)$

\begin{tabular}{ccccc}
\hline & & Idade & Série & Koppitz \\
Série & $r$ & $0,83^{* *}$ & & \\
Koppitz & $r$ & $-0,53^{* *}$ & $-0,67^{* *}$ & \\
B-SPG & $r$ & $-0,61^{* *}$ & $-0,69^{* *}$ & $0,82^{* *}$ \\
\hline
\end{tabular}

** Correlação é significante ao nível de 0,00।

Ao correlacionar a pontuação total das queixas agrupadas com as correções do Bender pela Koppitz e B-SPG, obtém-se um valor moderado positivo significativo em ambos os sistemas, embora mais relevante para Koppitz, sendo 0,34 $(p=0,002)$ e no B-SPG, $0,29(p=0,008)$. Porém ao analisar separadamente os tipos de queixas, os
Análise de Variância, obtendo-se os resultados de $[F(4,85)=10,14 ; p<0,000]$ para pontuação de Koppitz e $[F(4,85)=10,49 ; p<0,000]$ para os pontos de B-SPG. Esses dados demonstram que há diferença significativa entre as idades para os dois sistemas de correção. 
Tabela 5. Coeficiente de Correlação de Pearson entre os tipos de queixas e as pontuações dos sistemas de correção Koppitz e B-SPG (N=85)

\begin{tabular}{ccrr}
\hline & & Koppitz & \multicolumn{1}{c}{ B-SPG } \\
Comportamento & $r$ & $-0,29 * *$ & $-0,26^{* *}$ \\
Escolar & $r$ & $0,28^{* *}$ & $0,22^{*}$ \\
Emocional & $r$ & 0,11 & 0,11 \\
Queixa agrupada & $r$ & $0,34^{* *}$ & $0,29 * *$ \\
\hline
\end{tabular}

** Correlação é significante ao nível de 0.01 .

* Correlação é significante ao nível de 0.05 .

\section{Discussão}

Buscar parâmetros psicométricos nacionais para testes psicológicos, além de necessário para manter atualizadas as referências avaliativas, indica a preocupação e compromisso de profissionais com o caráter científico da psicologia. A escolha de um instrumento, dentre tantos, para ser alvo de pesquisa, revela a importância desse material para o pesquisador, por considerá-lo como um representante importante em sua categoria diagnóstica. Foi nessa perspectiva que houve a seleção do Teste Gestáltico Visomotor de Bender para o presente estudo. Sua relevância é confirmada pelo número de pesquisas no âmbito nacional e estrangeiro, assim como pela sua freqüência de uso clínico. Escolheu-se o sistema de Koppitz, por ser o mais citado, e o B-SPG por ser não apenas o único método de correção com parâmetros brasileiros, como de autores nacionais (Buckley, 1978; Noronha \& cols., 2002).

A comparação das médias entre os sistemas de correção fornece dados significativos para psicólogos e tende a colaborar para com o processo de avaliação psicológica. A média maior no sistema B-SPG é conseqüência da graduação de pontos de acordo com a qualidade do erro (atribuição de I a 3 pontos) e não apenas por sua presença (I ponto). A possibilidade de uma avaliação que possa identificar diferenças na qualidade das habilidades favorece a compreensão e
- planejamento da intervenção psicológica, como já preconizado por La Puente e Maciel Jr. (1984).

A análise das dificuldades visomotoras apresentadas pela amostra oferecem dados interessantes acerca da comparação dos dois sistemas de correção. No sistema Koppitz no critério integração (figura 4) e rotação (figura 2), a maior pontuação estão presentes, respectivamente, em figuras que não possuem a correção da distorção da forma. Esses dados tornamse importantes na medida em que revelam que a distorção da forma aparece como maior pontuador em todas as figuras nas quais está presente, evidenciando ser esse quesito o mais sensível para mensurar a percepção visomotora, o que corrobora as considerações de Sisto, Noronha e Santos (2006).

Considerando o objetivo de comparar o sistema Koppitz e o B-SPG, que se propõem a avaliar a maturidade visomotora, os dados são indicadores de evidência de validade convergente, pois se verificou que os instrumentos medem construtos similares, uma vez que houve correlação positiva forte e significativa entre ambos, porém não suficiente para serem consideradas formas paralelas. Ao se comparar pontuações totais com idades e séries, as correlações foram negativas altas e significativas, indicando que existe um aumento da maturidade visomotora com a idade e exigência de maior amadurecimento para promoção nas diversas séries do ensino acadêmico, corroborando os estudos de Gemignani e Chiari (2000) e Brannigan e Brunner (1989). 
Porém, os resultados desse estudo devem ser analisados com cautela uma vez que a amostra representa apenas crianças com queixas no seu desenvolvimento. Pelo fato de não se ter uma comparação com crianças de desenvolvimento normal, não se pode afirmar que os índices encontrados são discriminativos da maturidade percepto-motora. Nesse particular, os resultados obtidos a partir da correlação entre os sistemas e os tipos de queixas revelaram aspectos interessantes, já que exceto a emocional, as demais se correlacionaram significativamente, reafirmando os achados de Clawson (1980). Novas pesquisas que levam em consideração o tamanho das amostras parciais, e comparações com crianças de maturidade visomotora adequadas além de grupos específicos de distúrbios (Ferrante, 2004), poderão fornecer dados significativos para os dois sistemas de correção, visto que cada um tem suas especificidades. Outros estudos que promovam a comparação entre grupos heterogêneos são bem-vindos e tornam-se necessários.

\section{Referências}

Bandeira, R. D., \& Hutz, C. S. (1994). A contribuição dos testes DFH, Bender e Raven na predição do rendimento escolar na primeira série. Psicologia: Teoria e Pesquisa, IO(I), 59-72.

Brannigan, G. G., \& Brunner, N. A. (1989). The Modified Version of the Bender Gestalt Test for Preschool and Primary School Children. Brandon V T: Clinical Psychology Publishing.

Brannigan, G. G., \& Decker, S. L. (2003). Bender Gestalt II Bender Visual-Motor gestalt test. Second Edition. Riverside Publishing.

Brito, G. N. O., \& Santos, T. R. (1996). The Bender test for 5 to I5-year old Brazilian Children. Norms and Validity. Journal de Medicina e Biologia, 29, I5I3-15I8.

Buckley, P. D. (1978). The Bender Gestalt Test: A review of reported research with school-age subjects, 1966-1977. Psychology in the Schools, 15, 327-335.

Chan, P.W. (2000). Relationship of visual motor development and academic performance of young children in Hong
Kong assessed on the Bender Gestalt Test. Perceptual and Motor Skills, 90, 209-2I4.

Clawson, A. (1980). Bender Infantil: Manual de Diagnóstico Clínico. Porto Alegre,RS: Artes Médicas.

Conselho Federal de Psicologia (2006). Sistema de Avaliação dos Testes Psicológicos. Disponível em http:// www.pol.org.br. Acessado em julho/2006.

Dixon, J. L. (1998). Concurrent validity of the Koppitz BenderGestalt emotional indicators among womwn with mental retardion. Perceptual and Motor Skills, 86, 195- 197.

Ferrante, V. (2004). Scoring the Bender-Gestalt test using the Koppitz developmental system: Interrater reliability, item difficulty, and scoring implications. Revista Iberoamericana de Diagnostico y Evaluacion Psicologica, I8, I37-I59.

Germignani, E. Y. M. Y., \& Chiari, B. (2000). Escala de maturação do teste de Bender em um grupo de crianças deficientes auditivas. Pró-fono, I2(2), 49-53.

Koppitz, E. M. (1989). O teste Gestáltico Bender para crianças. Porto Alegre, RS: Artes Médicas.

La Puente, M., \& Maciel Jr., J. (1984). Procedimentos operacionais na avaliação do Teste bender infantil. Estudos de Psicologia, 3(4), 76-92.

Lesiak, J. (1984). The Bender Visual Motor Gestalt Test: implications for the diagnosis and prediction of reading achievement. Journal of School Psychology, 22, 39I-405.

Levin, M., \& González, N. (2004). Por quê hay ninos que no aprendem a escribir? Aportes de la evaluación psicológica. Revista Médica Tucumán, 10(2), 69-84.

Machado, M. C. L. (1978). Uso do teste de Bender para avaliar a organização percepto motora de escolares paulistas. Dissertação de Mestrado, PUC-SP, São Paulo.

Mazzeschi, C., \& Liz, A. (1999). The Bender-Gestalt test: Koppitz's Developmental Scoring System administered to two samples of Italian preschool and primary school children. Perceptual and Motor Skills, 88, I235-I 244.

Mitchell-Burns, J. A. (2000). Performance of children with and without learning disabilities on Canter's Background interference Produce and Koppitz's scoring system for the Bender test . Perceptual and Motor Skills, 90, 875-882.

Moura, M. L. S. (1982). Reconhecimento e reprodução de figuras do Teste Gestáltico Viso-Motor-Bender - um estudo evolutivo. Arquivos Brasileiros de Psicologia, 34(4), $50-61$. 
Nyfield, B., \& Palatano, F. (1998). Effects of serial position on Bender-Gestalt errors using Koppitz I's criteria. Psychology Reports, 83, 1227-1237.

Noronha, A. P. P., Oliveira, A. F., Cobêro, C., Paula, L. M., Cantalice, L. M., Guerra, P. B. C., Martins, R. M. M., \& Filizatti, R. (2002). Instrumentos psicológicos mais conhecidos por estudantes do sul de Minas Gerais. Avaliação Psicológica, I, I5I-I 58.

Pinelli Jr., B. \& Pasquali, L. (1990). Parâmetros Psicométricos do Teste Gestaltico Visomotor de Bender: um estudo empírico. Revista de Psicologia, I (2), 5 I-74.

Pinelli Jr., B., \& Pasquali, L. (199|/|992). Parâmetros psicométricos do Teste Gestáltico Viso-Motor de Bender: um estudo empírico. Revista de Psicologia, 9(I),5I-74.

Piotrowski, C. (1995). A review of the clinical and research use of the Bender-Gestalt Test. Perceptual and Motor Skills, $81,1272-1274$.

Raggio, D. J. (1999). Visuomotot perception in children with attention déficit hyperativity disorder-combined type. Perceptual and Motor Skills, 88, 448-450.
Sisto, F. F, Bueno, J. M. H., \& Rueda, F. J. M. (2003). Traços de personalidade na infância e distorção e integração de formas: um estudo de validade. Psicologia em Estudo, 8(I), 77-84.

Sisto, F. F., Noronha, A. P. P., \& Santos, A. A. A. (2004a). Distorção de forma no Teste de Bender: questionando seu critério de validade. Revista do Departamento de Psicologia da UFF, I6(2), 23-34.

Sisto, F. F., Santos, A. A. A, \& Noronha, A. P. P (2004b). Critério de integração do Teste Bender: explorando evidências de validade. Avaliação Psicológica,3(I), 28-34.

Sisto, F. F., Noronha, A. P. P., \& Santos, A. A. A. (2006). Bender Sistema de Pontuação Gradual B-SPG. Itatiba, SP: Programa de Pós-Graduação Stricto-Sensu em Psicologia da Universidade São Francisco. São Paulo,SP: Vetor.

Recebido em: 28/09/2006

Revisado em: 08/I2/2006

Aprovado em: 20/12/2006

Sobre as autoras

Ana Paula Porto Noronha (ana.noronha@saofrancisco.edu.br) é doutora em Psicologia e docente do Programa de Pós-Graduação Stricto Sensu em Psicologia da Universidade São Francisco. Bolsista de Produtividade em Pesquisa do CNPq.

Rosângela Maria De Carli Bueri Mattos (secpsicologia@bol.com.br) é mestre em Psicologia Clínica e docente da Universidade de Taubaté (SP) e do Centro Universitário de Itajubá - UNIVERSITAS.

Endereço para correspondência

Ana Paula Porto Noronha

Rua Alexandre Rodrigues Barbosa, 45, Centro

|325|-900, Itatiba, S.P. 